\title{
Theoretical investigation of the low frequency fundamental mechanism of the objective occlusion effect induced by bone-conducted stimulation
}

Kévin Carillo, ${ }^{1, a)}$ Olivier Doutres, ${ }^{1}$ and Franck Sgard ${ }^{2}$

${ }^{1}$ Department of Mechanical Engineering, École de technologie supérieure, Montréal, H3C 1K3, Canada

${ }^{2}$ Direction scientifique, IRSST, Montréal, H3A 3C2, Canada

\begin{abstract}
:
The objective occlusion effect induced by bone-conducted stimulation refers to the low frequency acoustic pressure increase that results from occluding the ear canal opening. This phenomenon is commonly interpreted as follows: the bone-conducted sound "leaks" through the earcanal opening and is "trapped" by the occlusion device. This instinctive interpretation misrepresents the fundamental mechanism of the occlusion effect related to the earcanal impedance increase and already highlighted by existing electro-acoustic models. However, these models simplify the earcanal wall vibration (i.e., the origin of the phenomenon) to a volume velocity source which, in the authors' opinion, (i) hinders an exhaustive comprehension of the vibro-acoustic behavior of the system, (ii) hides the influence of the earcanal wall vibration distribution, and (iii) could blur the interpretation of the occlusion effect. This paper analyzes, illustrates, and interprets the vibro-acoustic behavior of the open and occluded earcanal using an improved finite element model of an outer ear in conjunction with an associated electro-acoustic model developed in this work. The two models are very complementary to dissect physical phenomena and to highlight the influence of the earcanal wall vibration distribution, characterized here by its curvilinear centroid position, on the occlusion effect. @ 2020 Acoustical Society of America. https://doi.org/10.1121/10.0001237
\end{abstract}

(Received 22 July 2019; revised 24 April 2020; accepted 24 April 2020; published online 13 May 2020)

[Editor: Christopher Shera]

Pages: $3476-3489$

\section{INTRODUCTION}

The term occlusion effect (OE) is commonly used to describe an increase in the auditory perception of boneconducted (BC) sound that results from occluding the earcanal (EC) opening. In everyday life, the $\mathrm{OE}$ is often perceived as the increased sensation of the BC part of one's own physiological noises ${ }^{1}$ (e.g., one's own voice, breathing, chewing, heartbeat), which is most significant for low frequencies (LF; $<1 \mathrm{kHz}$ ). The $\mathrm{OE}$ is deemed to be a notable source of discomfort to workers wearing shallowly inserted passive occlusion devices such as earplugs ${ }^{2}(\mathrm{EP})$. The $\mathrm{OE}$ also affects hearing aid users' comfort ${ }^{3}$ but can be greatly reduced using open-fittings. ${ }^{4}$ The deep-fitting of the occlusion device in the bony part of the EC is also known to reduce the OE (Ref. 5) but seems associated with wearers' discomfort. ${ }^{6,7}$ The BC sound travels to the cochlea through different pathways. ${ }^{8-11}$ The outer ear pathway corresponds to the sound pressure generated in the EC cavity due to the vibration of the EC wall, which constitutes the source of the OE. At LF, the outer ear pathway is negligible when the EC is open ${ }^{12,13}$ but dominates when it is occluded. ${ }^{12}$ The OE is thus objectively characterized by an acoustic pressure increase in the occluded EC at LF. ${ }^{14-16}$ This objective description of the $\mathrm{OE}$ is the one used in this paper.

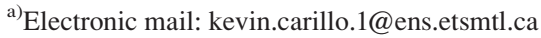

In the literature, the $\mathrm{OE}$ is commonly interpreted as follows: the BC sound generated by the EC wall "escapes" through the EC opening (i.e., open EC entrance), whereas it is "trapped" in the occluded case. ${ }^{6,17,18}$ The BC sound is expected to take the path of least "resistance." 19 Sometimes, authors refer to the sound pressure generated by the EC wall $^{7,20}$ or to the "sound vibration" imposed by the EC wall. ${ }^{4}$ In many cases, the EC opening is interpreted as a "leak" for the sound energy which is presumed to be "trapped" in the occluded EC. ${ }^{21-25}$ Therefore, sound waves generated by the EC wall are assumed to be mainly transmitted in the surrounding environment in the open case. On the contrary, they are expected to be mainly reflected by the occlusion device towards the tympanic membrane (TM), leading to an enhanced acoustic pressure in the occluded EC. $^{26}$ These multiple interpretations, found either in experimental or modeling studies in both hearing aid and hearing protection fields, are ambiguous because they describe the OE using several physical variables, concepts, and an instinctive vocabulary. Furthermore, the way the phenomenon is interpreted could influence the solutions sought to reduce it. These interpretations thus must be clarified and OE models can be helpful for this purpose.

In order to investigate the $\mathrm{OE}$, different models have been elaborated in the past. ${ }^{15,16,27-32}$ Lumped elements models based on electro-acoustic (EA) analogy $15,16,27,28$ are historically the first OE models. These models account for 
the air within the EC cavity as acoustic masses, compliances, and resistances and have highlighted the fundamental mechanism of the $\mathrm{OE}$ as the increase of the EC cavity acoustic impedance seen by the cavities' vibrating walls due to the occlusion. However, the spatially distributed EC wall vibration (i.e., the source of the $\mathrm{OE}$ ) is idealized as one $^{15,28,29}$ or two ${ }^{16}$ volume velocity source(s) concentrated at a chosen position in the EC. Due to the cramped and tortuous aspects of the human EC, amplitude and position of the source(s) are difficult to assess experimentally and are generally assumed or adjusted based on sound pressure measurements in open and occluded EC. This prevents any analysis of the influence of the EC wall vibration amplitude and, in particular, distribution, though it is the source of the OE. In addition, the EC cavity is commonly simplified to one $^{15,28,29}$ or two ${ }^{16}$ uniform cylindrical duct(s), considering that the acoustic wavelength is much larger than the EC dimensions at LF. To overcome these limitations, finite element (FE) models ${ }^{30-32}$ of the OE can be used since they make it possible to account for a precise geometry, material properties, boundary conditions, and stimulation. However, FE models are limited by the knowledge of the corresponding inputs, which can be very difficult to characterize experimentally. Recently, Brummund et $a l^{30}$ developed a three-dimensional (3D) FE model of a "realistic" outer ear in which the set of equivalent loading and boundary conditions is debatable. Indeed, in order to mimic the placement of a standard bone transducer, a normal force was applied directly on the mastoid process while the remaining surface of the truncated geometry was fixed. ${ }^{30}$ This set induces a compression motion in the temporal bone, which is rather expected to behave as a rigid body at LF. ${ }^{12}$ In addition, this set forces the vibration to travel from the bony tissue to the soft and skin tissues while the latter should be rather primarily excited by the bone transducer. Despite these limitations, this model has been shown to provide the slope of the OE at shallow/medium insertion ${ }^{30}$ measured on 20 subjects by Stenfelt and Reinfeldt. ${ }^{16}$ Based on this model, Brummund et $a l .{ }^{30}$ investigated the fundamental mechanism of the $\mathrm{OE}$ using an acoustic power balance approach. They pointed out that an acoustically rigid occlusion of the EC entrance increases the resistance (real part) of the EC cavity acoustic impedance seen by its wall compared to the open case. ${ }^{30}$ This resistance governs the acoustic power injected into the EC cavity by its wall. ${ }^{30}$ However, Brummund et al. ${ }^{30}$ assumed no visco-thermal dissipation within the EC cavity, although it is higher than the acoustic power radiated at the open EC entrance at $\mathrm{LF}^{33}$ and could have underestimated the resistance of the open EC. In addition, the acoustic power balance approach only describes the acoustic energy transfer but does not detail the vibro-acoustic (i.e., acoustic pressures and particle velocities) behavior that explains this transfer. The use and limits of this approach are thus revisited in this work.

Both EA and FE models are useful to explain the fundamental mechanism of the OE. However, the source of the $\mathrm{OE}$, namely, the vibration of the EC wall, is overly simplified in EA models and too intricate in FE models. The influence of the EC wall vibration is thus hidden in both models. In addition, these models have not been used to fully detail physical phenomena occurring in both open and occluded EC under a BC stimulation. In the authors' opinion, taking these steps (i.e., investigating the influence of the EC wall vibration and detail physical phenomena in the EC cavity) is necessary to accurately revisit our understanding of the OE and the role of its source, to clarify the ambiguity that surrounds the interpretation of the $\mathrm{OE}$ and to ultimately open new ways to mitigate this phenomenon. For this purpose, the FE model of a "realistic" outer ear proposed by Brummund et al. ${ }^{30}$ is revisited, improved, and used in conjunction with an associated EA model developed in this work. The occlusion is simplified to an infinite impedance defined at the EC entrance to focus on the fundamental mechanism of the OE. In other words, the intricate influence of the occlusion device $\mathrm{s}^{30-32,34,35}$ is not taken into account.

The originality of this work is threefold. First, the FE model is used to thoroughly analyze and illustrate the vibroacoustic behavior of both open and occluded EC at LF from $100 \mathrm{~Hz}$ to $1 \mathrm{kHz}$. Second, all inputs of the EA model presented here are derived from the FE model. In particular, the volume velocity source characteristics are here directly related to the EC wall vibration field. The EA model is thus useful to clarify and interpret in a simplified way physical phenomena involved in the EC as well as the influence of the EC wall vibration distribution on the OE. Results computed using both FE and EA models are presented, compared, and discussed. Third, common interpretations of the $\mathrm{OE}$ are discussed in light of the results presented here in open and occluded cases.

\section{MODELS OF THE OCCLUSION EFFECT}

An implicit harmonic temporal dependency is taken into account by the term $e^{j \omega t}$, where $j$ is the complex number such as $j^{2}=-1, \omega$ is the circular frequency such that $\omega=2 \pi f, f$ denotes the frequency, and $t$ the time. An arbitrary space and time dependent physical variable $h(\underline{x}, t)$ is written in the complex form such that

$$
h(\underline{x}, t)=\Re\left[\hat{h}(\underline{x}) e^{j \omega t}\right],
$$

where $\underline{x}=\left(x_{1}, x_{2}, x_{3}\right)$ denotes the position vector of the point of interest in the 3D space, $\hat{h}$ is the complex amplitude and $\Re[\cdot]$ takes the real part of its argument. Solving for $\hat{h}$ is equivalent to solving for $h$. In addition, $\hat{h}^{*}$ is the complex conjugate, $|\hat{h}|$ is the modulus and $\langle\hat{h}\rangle$ is the surface averaged value. In the EA model, all vibro-acoustic variables are spatial averages and $\hat{h}=\langle\hat{h}\rangle$.

\section{A. Finite element model of the outer ear}

\section{Geometry and materials}

The FE model of the outer ear developed by Brummund et al. $^{30}$ and used in this study is illustrated in Fig. 1(a). The 

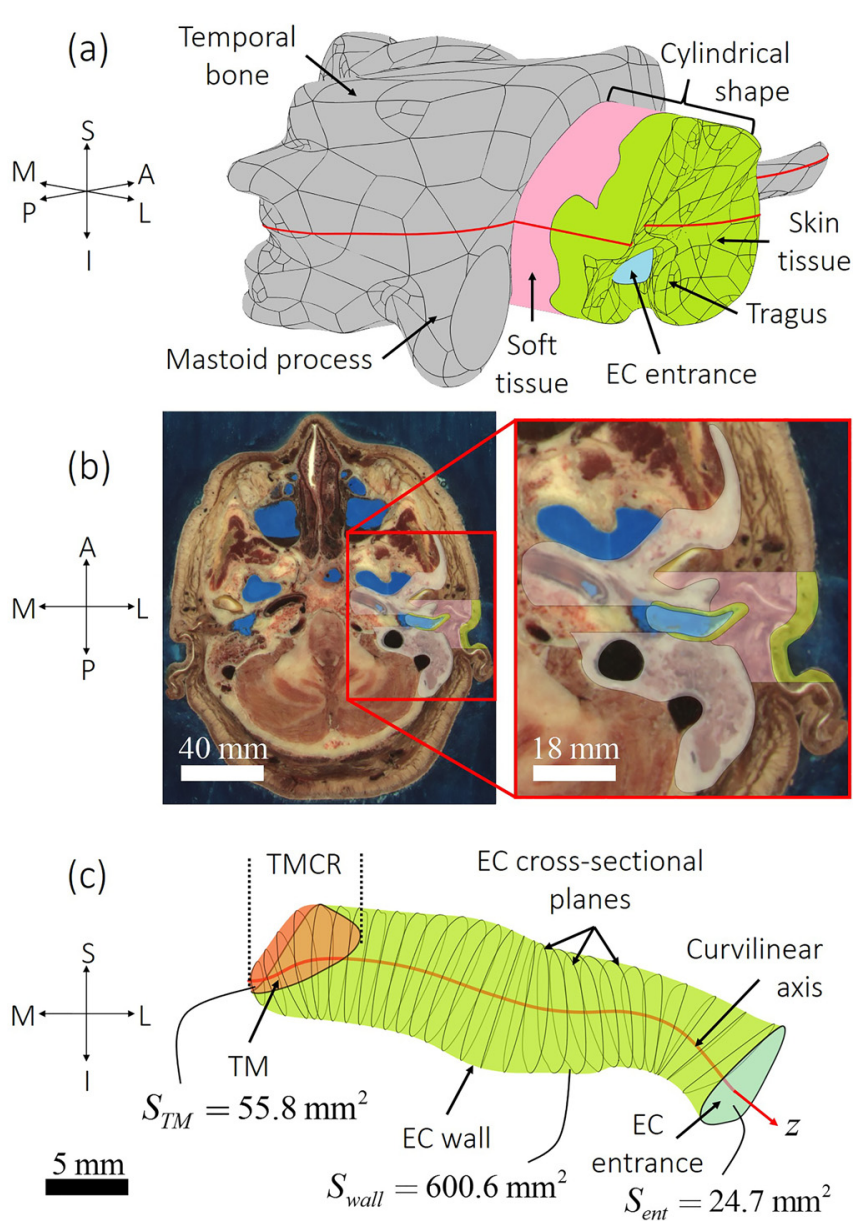

FIG. 1. (Color online) (a) 3D FE model of an outer ear (Ref. 30), (b) sectional view in the horizontal plane [specified by a red line in (a)] superimposed on the corresponding cryosection image from the Visible Human Project $^{(}$, and (c) EC cavity alone. The coordinate system refers to superior $(\mathrm{S})$, inferior (I), posterior (P), anterior (A), medial (M), and lateral (L).

geometry was obtained from cross-sectional cryosection images of a female cadaver from the Visible Human Project $^{\circledR}$. Figure 1(b) presents a sectional view in the horizontal plane of the FE model superimposed on the corresponding cryosection image. In the FE model, the EC cavity is embedded into a circular cross section cylinder (mediallateral axis) with a diameter of $27 \mathrm{~mm}$ [see Fig. 1(a)]. This reduces the computation time compared to an entire head model. ${ }^{36}$ This model distinguishes the skin (including the fat) from other soft tissues (including cartilage and muscle) and it includes the entire temporal bone [the gray part of Fig. 1(a)]. More recent studies rather separate cartilage from soft tissues (including skin, fat and muscle). ${ }^{36,37}$ In the current model, the pinna and the middle and inner ears are not fully modeled but are accounted for by estimates of the acoustic impedance associated with sound flow through these structures (see Sec. II A 2). A complete view of the EC cavity from the TM to the EC entrance is presented in Fig. 1(c). The EC curvilinear axis $z$ [see Fig. 1(c)], also called middle or center axis in the literature, is computed using an iterative method developed by Stinson and Lawton. ${ }^{38}$ In this work, the EC entrance plane is defined normal to the EC curvilinear axis rather than in a sagittal plane. ${ }^{30}$ The EC shape represented as a radius function $r_{E C}(z)$ (assuming circular cross-sections) is displayed in Fig. 2. Compared to 15 radius functions presented by Hudde and Engel, ${ }^{39}$ the current model includes a constriction close to the EC entrance. In addition, the current EC curvilinear length $\left(l_{E C}=34.7 \mathrm{~mm}\right)$ is higher than the average EC length (known to be around $27 \mathrm{~mm}$ ) while the tympanic membrane coupling region (TMCR) length (close to $8 \mathrm{~mm}$ ) is similar. ${ }^{39}$

Solid domains are considered isotropic linear elastic solids under the small deformations' assumption. Their mechanical properties come from literature data (except structural loss factors that have been assumed ${ }^{30}$ ) and are summarized in Table II. While the air within the EC cavity was considered a perfect compressible fluid by Brummund et al., ${ }^{30}$ it is here rather modeled as a Navier-StokesFourier compressible fluid to account for visco-thermal dissipation. ${ }^{40,41}$ Classic values of ambient air properties are used and given in Table III.

\section{Boundary conditions and model limitations}

In the authors' opinion, the main limitation of the current FE model developed by Brummund et al. ${ }^{30}$ comes from its truncated geometry [see Fig. 1(a)]. As exposed in Sec. I, this model requires the imposition of a set of equivalent loading and boundary conditions which are particularly tricky to define since the truncated geometry is supposed to be surrounded by biological tissues [see Fig. 1(b)]. The set chosen by Brummund et al. ${ }^{30}$ results in a vibration pattern concentrated approximately at the EC mid-length (see Appendix B) near the transition between soft and bony tissues while this vibration is rather expected to be concentrated in the EC cartilaginous part close to the EC entrance. ${ }^{12,16}$ In this paper, another set is chosen in order to be more consistent with realistic loading and boundary conditions and to reproduce a "plausible" vibration pattern (see Sec. III A), even though the latter has never been directly measured due to experimental limitations nor studied numerically. A bone transducer stimulation applied on the mastoid process is expected to induce a compression motion in the skin and soft tissues which then stimulate the temporal bone. Therefore, skin and soft tissues are here excited in a simplified way by a uniform normal velocity field

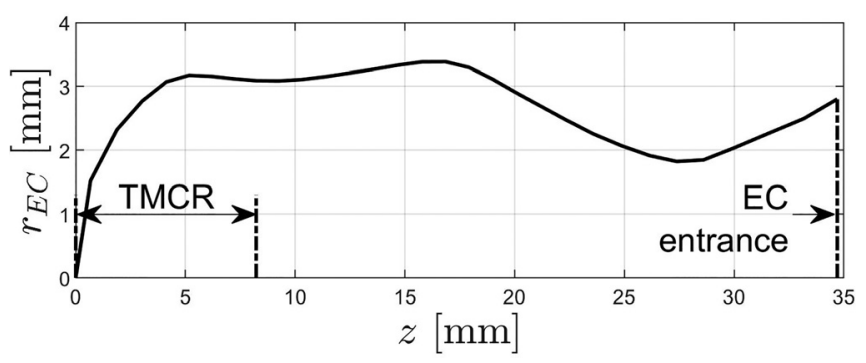

FIG. 2. EC radius $r_{E C}(z)$ (assuming circular cross-sections) as a function of the curvilinear axis $z$. 
$V_{n}(\underline{x}, t)=\Re\left[A(\underline{x}) e^{j \omega t}\right]$ applied to their circumferential surface. The amplitude $A(\underline{x})=10^{-5} \mathrm{~m} \mathrm{~s}^{-1}$ is chosen to produce realistic sound pressure levels in both open and occluded EC (see Sec. IIID). The temporal bone external boundary and the skin in contact with the surrounding environment (not modeled $^{30}$ ) are free. Continuity of stress vectors and displacements is assumed between solid domains. Since the EC wall vibration distribution depends on the chosen set of loading and boundary conditions, the influence of various sets (summarized in Appendix B) on the OE is studied (see Sec. IIIF). Due to its truncation, the current model is not used to study the BC sound transmission through the head or the outer ear for a specific individual but rather to detail general physical phenomena in the EC cavity.

Acoustic boundary conditions applied to the EC cavity are similar to those used by Brummund et al. ${ }^{30}$ A locally reacting acoustic impedance boundary condition is specified on the TM of surface $S_{T M}$ [see Fig. 1(c)]. The impedance associated with sound-induced motion of the TM is referred to as $\hat{Z}_{T M}$ and defined by Shaw and Stinson's model ${ }^{15,42,43}$ which compares well to measurement data at $\mathrm{LF}^{44}$ An acoustic impedance $\hat{Z}_{\text {ent }, k}$, where $k \in\{$ open, occluded $\}$, is also defined at the EC entrance of surface $S_{\text {ent }}$ [see Fig. $1(c)]$. In the open case, this impedance is equal to the radiation impedance $\hat{Z}_{\text {rad }}$ of a baffled circular piston of the same surface (see Appendix C). At LF, this impedance is a reasonable approximation of that measured if the EC entrance plane is chosen not too "inside" the EC (portion of the EC included) nor too "outside" (acoustic wave no longer plane). ${ }^{39}$ In the occluded case considered here, the EC entrance has an infinite impedance instead of a real physical occlusion device. $^{30}$

\section{Finite element modeling}

In the coupled elasto-acoustic (visco-thermal) model, the coupling at the EC wall/cavity boundary implies the continuity of velocity vectors and stress vectors. The EC wall is assumed isothermal since the human body is considered at constant temperature. Adiabatic thermal couplings are assumed at the EC entrance and the TM since negligible heat transfers are expected. The visco-thermal acoustics model requires lots of computational resources due to the number of variables and the refinement of the mesh at the EC wall boundary. This model is thus only used to accurately illustrate physical phenomena in the 3D EC cavity at $100 \mathrm{~Hz}$ (see Fig. 4). Vibro-acoustic indicators presented in Sec. II A 4 are computed over the whole frequency range of interest using a low reduced frequency (LRF) model $^{45}$ applied to the EC cavity. In the LRF model, viscous and thermal losses are distributed in the bulk of the fluid through a complex wavenumber. ${ }^{41,45}$ The coupling at the EC wall boundary is thus simplified to the continuity of normal component velocity vectors and normal component stress vectors. ${ }^{46}$ The radius used for the LRF model corresponds to the equivalent EC radius function $r_{E C}(z)$ (see Fig. 2). Both coupled elasto-acoustic models are meshed according to a criterion of at least six 10-nodded tetrahedral elements per wavelength at $1 \mathrm{kHz}$ and solved using COMSOL Multiphysics 5.5 (COMSOL ${ }^{\circledR}$, Sweden). The meshes of the LRF and the visco-thermal FE models consist of 613452 and 1331740 elements, respectively, and achieve convergence.

\section{Vibro-acoustic indicators}

When submitted to the chosen mechanical excitation (see Sec. II A 1), the EC wall vibrates and imposes to the EC cavity a volume velocity defined by $\hat{q}_{\text {wall }}=\left\langle\hat{v}_{n \text {,wall }}\right\rangle S_{\text {wall }}$ with $\left\langle\hat{v}_{n, \text { wall }}\right\rangle$ the surface averaged EC wall normal velocity and $S_{\text {wall }}$ the EC wall surface. According to Brummund et al., ${ }^{30}\left\langle\hat{v}_{n, \text { wall }}\right\rangle$ is only slightly reduced $(<1 \mathrm{~dB})$ at LF by the EC cavity acoustic load change between open and occluded cases (confirmed but not shown here). Since the occlusion is defined at the EC entrance, $\hat{q}_{\text {wall }}$ is thus approximately equal in both open and occluded cases. Volume velocities passing through the TM and the EC entrance (zero in the occluded case due to the acoustically rigid occlusion) are referred to as $\hat{q}_{T M, k}$ and $\hat{q}_{\text {ent }, k}$, respectively, and computed similarly to $\hat{q}_{\text {wall }, k}$. The EC wall vibration is not expected to be homogeneous but rather non-uniformly distributed over the EC wall surface. This distribution is here characterized by the centroid position $\underline{x}_{c}$ of the EC wall normal velocity in the $3 \mathrm{D}$ space such that

$$
\underline{x}_{c}=\frac{\int_{S_{\text {wall }}} \underline{x}\left|\hat{v}_{n, \text { wall }}(\underline{x})\right| \mathrm{d} S}{\int_{S_{\text {wall }}}\left|\hat{v}_{n, \text { wall }}(\underline{x})\right| \mathrm{d} S} .
$$

The normal projection of the centroid position $\underline{x}_{c}$ on the EC curvilinear axis $z$ defines the centroid curvilinear position $l_{c}$ of the EC wall normal velocity from the TM.

The surface averaged acoustic pressure $\left\langle\hat{p}_{\text {wall }, k}\right\rangle$ generated by the EC wall is governed by the EC acoustic impedance $\hat{Z}_{E C / \text { wall }, k}$ seen by the EC wall and defined by

$$
\hat{Z}_{E C / \text { wall }, k}=\frac{\left\langle\hat{p}_{\text {wall }, k}\right\rangle}{\hat{q}_{\text {wall }}} .
$$

The OE is then computed using the surface averaged TM acoustic pressure $\left\langle\hat{p}_{T M, k}\right\rangle$ such that

$$
O E=20 \log _{10}\left(\left|\frac{\left\langle\hat{p}_{T M, \text { occl }}\right\rangle}{\left\langle\hat{p}_{T M, \text { open }}\right\rangle}\right|\right) .
$$

The OE is commonly interpreted in terms of acoustic energy (see Sec. I). The transfer of acoustic energy per unit time in the EC cavity is studied here as time averaged over one period $T=1 / f$ and is referred to as the time averaged acoustic power. The time averaged acoustic power flowing through the EC wall $\left(\bar{W}_{\text {wall }, k}\right)$, the TM $\left(\bar{W}_{T M, k}\right)$ and the EC entrance $\left(\bar{W}_{\text {ent }, k}\right.$, zero in the occluded case due to the acoustically rigid occlusion) is defined by 


$$
\bar{W}_{m, k}=\left|\int_{S_{m}} \bar{I}_{k} \cdot \underline{n}_{m} \mathrm{~d} S\right|
$$

with $\quad \bar{I}_{k}=\frac{1}{2} \Re\left[\hat{p}_{k} \times \underline{\hat{v}}_{k}^{*}\right]$ the active acoustic intensity vector, ${ }^{47} \hat{p}_{k}$ the complex acoustic pressure, $\underline{\hat{v}}_{k}$ the complex acoustic particle velocity vector, $\underline{n}_{m}$ the inward normal vector and $m \in\{$ wall, TM, ent $\}$. The acoustic power $\bar{W}_{\text {diss }, k}$ dissipated by visco-thermal effects in the EC cavity is then obtained from the acoustic power balance such as $\bar{W}_{\text {diss }, k}=\bar{W}_{\text {wall }, k}-\bar{W}_{T M, k}-\bar{W}_{\text {ent }, k}$.

\section{B. Electro-acoustic model of the earcanal}

\section{Electro-acoustic model}

The present EA model illustrated in Fig. 3(a) is associated with the 3D FE model from which its inputs are derived (see Sec. II B 3). The EC is considered as a circular crosssectional duct of length $l_{E C}$ and varying radius $r_{E C}(z)$. The vibration of the EC wall is accounted for as an ideal source $\mathrm{Q}$ of volume velocity $\hat{q}_{\text {wall }}$ concentrated at a distance $l_{c}$ from the TM. The EC is schematically divided into two sections: (i) the downstream section defined from the $\mathrm{TM}$ to the source and (ii) the upstream section defined from the source to the EC entrance. In both open [see Fig. 3(b)] and occluded [see Fig. 3(c)] cases, the model of Shaw and Stinson $^{42}$ is used to define the TM acoustic impedance $\hat{Z}_{T M}$. Furthermore, the downstream section is governed at LF by its compressibility effect of acoustic compliance $C_{d}$ in parallel with an acoustic resistance $R_{d, t h}$ representing
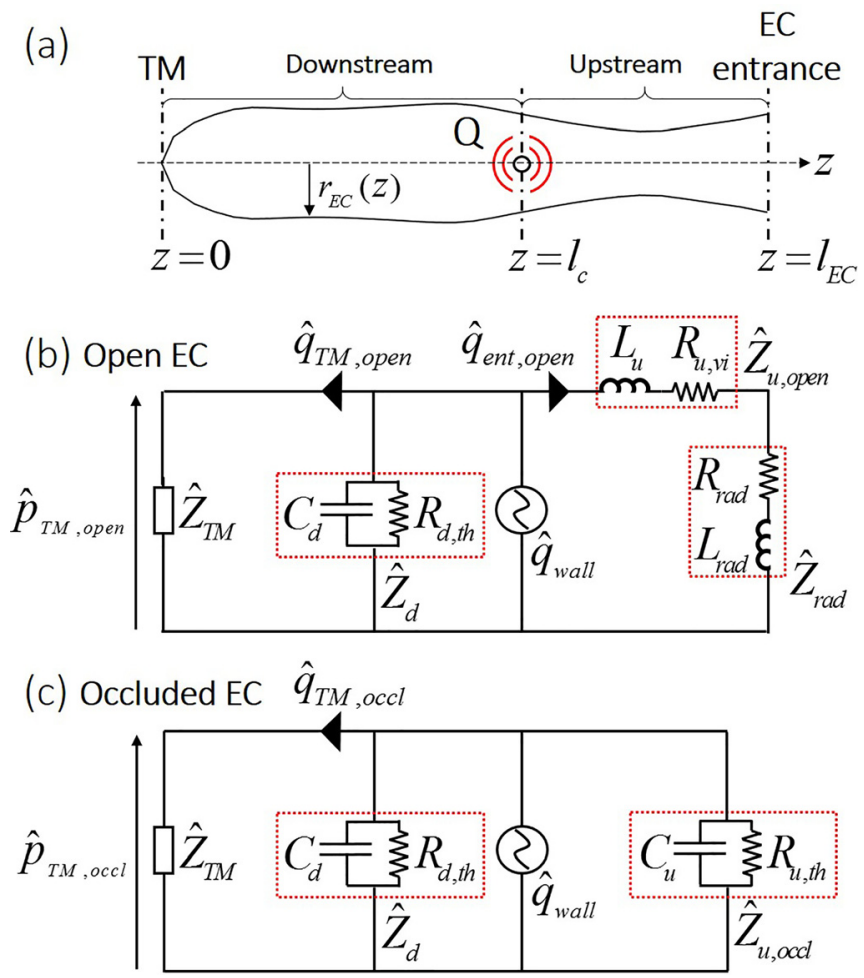

FIG. 3. (Color online) (a) EC geometry of the EA model, (b) open, and (c) occluded by an infinite impedance defined at the EC entrance. thermal losses so that its acoustic impedance is equal to $\hat{Z}_{d}=\left[R_{d, t h}^{-1}+j \omega C_{d}\right]^{-1}$. In the open case, the EC upstream section is controlled at LF by its inertia effect represented by an acoustic mass $L_{u}$ in series with an acoustic resistance $R_{u, v i}$ representing viscous losses so that its acoustic impedance is equal to $\hat{Z}_{u, o p e n}=R_{u, v i}+j \omega L_{u}$. The EC opening is characterized by the acoustic impedance $\hat{Z}_{\text {rad }}=R_{\text {rad }}+j \omega L_{\text {rad }}$ of a baffled circular piston of radius $r_{\text {ent }} . R_{\text {rad }}$ is the acoustic radiation resistance representing the dissipation in the surrounding environment while $L_{\text {rad }}$ is the acoustic radiation mass representing the inertia effect of the surrounding environment. In the occluded case, the acoustic impedance $\hat{Z}_{u, o c c l}=\left[R_{u, t h}^{-1}+j \omega C_{u}\right]^{-1}$ of the EC upstream section is governed at LF by its compressibility effect similarly to $\hat{Z}_{d}$. Table I summarizes the calculation $^{47}$ of aforementioned acoustic masses, compliances, and resistances.

\section{Computation of vibro-acoustic indicators}

The EA model is used to provide explicit expression of some vibro-acoustic indicators computed using the FE model. The acoustic impedance $\hat{Z}_{E C / Q, k}$ of the EC seen by the source $\mathrm{Q}$ is computed as the downstream and upstream sections in parallel [see Figs. 3(b) and 3(c)]. The acoustic impedance of the EC downstream section is defined in both open and occluded cases by $\hat{Z}_{E C / Q, d}=\left[\hat{Z}_{d}^{-1}+\hat{Z}_{T M}^{-1}\right]^{-1}$. The EC upstream section acoustic impedance is given in the open case by $\hat{Z}_{E C / Q, u, \text { open }}=\hat{Z}_{u, \text { open }}+\hat{Z}_{\text {rad }}$ and in the occluded case by $\hat{Z}_{E C / Q, u, o c c l}=\hat{Z}_{u, o c c l}$. In the $\mathrm{LF}$ range of interest (i.e., $100 \mathrm{~Hz}$ to $1 \mathrm{kHz}), \hat{Z}_{d}$ is dominated by $C_{d}$, $\hat{Z}_{u, \text { open }}$ by $L_{u}, \hat{Z}_{\text {rad }}$ by $L_{\text {rad }}$ and $\hat{Z}_{u, o c c l}$ by $C_{u}$ (not shown here for the sake of conciseness). In addition, below $\approx 500 \mathrm{~Hz}$, the TM acoustic impedance is dominated by the compliance $C_{T M}$ of the tympanic cavity volume ${ }^{29,48}\left(\approx 0.78 \mathrm{~cm}^{3}\right)$. Therefore, the EC acoustic impedance can be approximated at LF (see Sec. III C) in the open case to

$$
\hat{Z}_{E C / Q, o p e n} \approx j \omega\left(L_{\text {rad }}+L_{u}\right)
$$

and in the occluded case to

$$
\hat{Z}_{E C / Q, o c c l} \approx\left[j \omega\left(C_{u}+C_{d}+C_{T M}\right)\right]^{-1} .
$$

The TM acoustic pressure $\hat{p}_{T M, k}$ is equal to that generated by the source [see Figs. 3(b) and 3(c)] so that

TABLE I. Calculation of the EA model localized constants. $C_{u}$ and $R_{u, t h}$ are computed similarly to $C_{d}$ and $R_{d, t h}$, respectively, replacing the interval $\left[0, l_{c}\right]$ by $\left[l_{c}, l_{E C}\right]$.

\begin{tabular}{cc}
\hline$C_{d}=\int_{0}^{l_{c}} \frac{\pi r_{E C}^{2}}{\rho_{0} c_{0}^{2}} \mathrm{~d} z$ & $R_{d, t h}=\left[\int_{0}^{l_{c}}(\gamma-1) \frac{\pi r_{E C}}{\rho_{0} c_{0}^{2}} \sqrt{\frac{2 \lambda \omega}{\rho_{0} C_{p}}} \mathrm{~d} z\right]^{-1}$ \\
$L_{u}=\int_{l_{c}}^{l_{E C}} \frac{\rho_{0}}{\pi r_{E C}^{2}} \mathrm{~d} z$ & $R_{u, v i}=\int_{l_{c}}^{l_{E C}} \frac{\sqrt{2 \rho_{0} \omega \mu}}{\pi r_{E C}^{3}} \mathrm{~d} z$ \\
$L_{\text {rad }}=\frac{8 \rho_{0}}{3 \pi^{2} r_{\text {ent }}}$ & $R_{\text {rad }}=\frac{\omega^{2} \rho_{0}}{2 \pi c_{0}}$ \\
\hline
\end{tabular}




$$
\hat{p}_{T M, k}=\hat{Z}_{E C / Q, k} \times \hat{q}_{\text {wall }} .
$$

Substituting Eq. (8) in Eq. (4), the OE is written as

$$
O E=20 \log _{10}\left(\mid \frac{\hat{Z}_{E C / Q, o c c l}}{\hat{Z}_{E C / Q, \text { open }} \mid}\right) .
$$

At LF, substituting Eqs. (6) and (7) in Eq. (9), the OE can be approximated (see Sec. III F) to

$$
O E \approx 20 \log _{10}\left(\left[\omega^{2} \times\left(L_{r a d}+L_{u}\right) \times\left(C_{u}+C_{d}+C_{T M}\right)\right]^{-1}\right) .
$$

The equivalent of the acoustic power $\bar{W}_{\text {wall }, k}$ passing through the EC wall in the FE model is the acoustic power flowing from the source $\mathrm{Q}$ to the EC defined in the EA model by

$$
\bar{W}_{Q, k}=\frac{1}{2} \Re\left[\hat{Z}_{E C / Q, k}\right] \times\left|\hat{q}_{\text {wall }}\right|^{2} .
$$

The EC cavity acoustic resistance $\Re\left[\hat{Z}_{E C / Q, k}\right]$ represents the capability of the EC cavity (including the entrance and the TM) to dissipate/transmit acoustic energy. Equations (6) and (7) thus cannot be used in Eq. (11) since they neglect acoustic resistances.

\section{Inputs from the finite element model}

In the EA model, the EC has the same radius function [i.e., $l_{E C}, r_{E C}(z)$ and $r_{e n t}=r_{E C}\left(l_{E C}\right)$ ] as in the 3D FE model (see Table I). The ideal volume velocity source $\mathrm{Q}$ is assumed to be concentrated at the curvilinear position $l_{c}$ of the EC wall normal velocity centroid computed using the FE model (see Sec. II A 4). This assumption has been proposed by the authors ${ }^{49}$ and is evaluated in Sec. III F for several EC wall vibration patterns induced by various mechanical loading and boundary conditions imposed to the FE model (see Appendix B). Using the set of loading and boundary conditions described in Sec. II A 2, the position $l_{c}$ computed using the FE model does not significantly vary at LF (see Sec. III A). The value of $l_{c}$ at $100 \mathrm{~Hz}$ is thus used in the EA model for the whole frequency range. The volume velocity $\hat{q}_{\text {wall }}$ is computed using the FE model (see Sec. II A 4).

\section{RESULTS AND DISCUSSIONS}

\section{A. Earcanal wall velocity}

The normal component of the EC wall velocity is of great interest since it is transferred to "acoustic particles" in its vicinity. The curvilinear centroid position $l_{c}$ and the amplitude of the EC wall normal velocity computed at $100 \mathrm{~Hz}$ using the FE model are illustrated in Fig. 4(a). The centroid position characterizes the distribution of normal velocity over the EC wall (see Sec. II A 4). This distribution is here concentrated at $100 \mathrm{~Hz}$ at a curvilinear distance $l_{c}=11.1 \mathrm{~mm}$ from the TM [see Fig. 4(a), left side] which corresponds to the EC cartilaginous part. The location of $l_{c}$ varies by less than $1 \mathrm{~mm}$ as frequency increases from $100 \mathrm{~Hz}$ to $1 \mathrm{kHz}$. Thus, Fig. 4(a) (right side) is representative of the FE model EC wall vibration pattern up to $1 \mathrm{kHz}$. As mentioned in Sec. II A 2, this vibration pattern depends on the set of loading and boundary conditions applied to the FE model and has been chosen in order to exhibit a vibration pattern in qualitative agreement with experimental findings. ${ }^{12,16}$ The chosen set is a simplification imposed by the truncated nature of the current model. In particular, the uniform normal velocity field applied on the circumferential boundary of soft and skin tissues is likely to be non-uniform in amplitude, phase and direction. The lack of quantitative experimental data do not make it possible yet to precisely compare the FE model vibration distribution to that induced by a bone transducer in an in vivo EC. However, by applying various sets of loading and boundary conditions (see Appendix B), the current model is useful to highlight how the $\mathrm{EC}$ wall vibration distribution could influence the $\mathrm{OE}$ (see Sec. III F).

\section{B. Acoustic particle velocity and volume velocity transfer in the earcanal cavity}

The transfer of the EC wall velocity in the EC cavity is illustrated in Fig. 4(b) which displays the instantaneous (at $t \in\{T / 8,5 T / 8\}$ ) acoustic particle velocity vectors and amplitude computed in both open and occluded cases at $100 \mathrm{~Hz}$ using the FE model. The Eulerian specification of the acoustic field is used here. This means that vector fields represent the velocity of "acoustic particles" passing through a position $\underline{x}$ at a time $t$. At $t=T / 8$, the acoustic particle velocity is mainly oriented towards the EC entrance in the open case (left side) and towards the TM in the occluded case (right side) due to the compression motion of the EC wall. In the half-period that follows (at $t=5 T / 8$ ), the acoustic particle velocity behaves in the exact opposite direction since $h(\underline{x}, t+T / 2)=-h(\underline{x}, t)$ in harmonic regime [see Eq. (1)]. In the open case, the amplitude of the acoustic particle velocity is maximum in the constriction part of the current EC close to the opening and minimum in the TMCR. The gradient of acoustic particle velocity tangential to the EC wall is thus maximum in the region of this constriction. In the occluded case, the acoustic particle velocity amplitude is rather homogeneous except at the EC entrance where it is zero due to the acoustically rigid occlusion considered here. Note that a real occlusion device would impose a non-zero volume velocity to the occluded EC cavity.

The transfer of acoustic particle velocity in the EC cavity is now investigated by computing the volume velocity passing through the EC entrance and TM surfaces using both FE and EA models in Fig. 5. Results computed by both models are in good agreement. In the open case, the volume velocity passing through the EC entrance is $\approx 16$ to $52 \mathrm{~dB}$ higher than that passing through the TM. The volume velocity passing through the EC opening is almost equal to that imposed by the EC wall (not shown here). This corroborates the observation made in Fig. 4(b) for the open case (left 


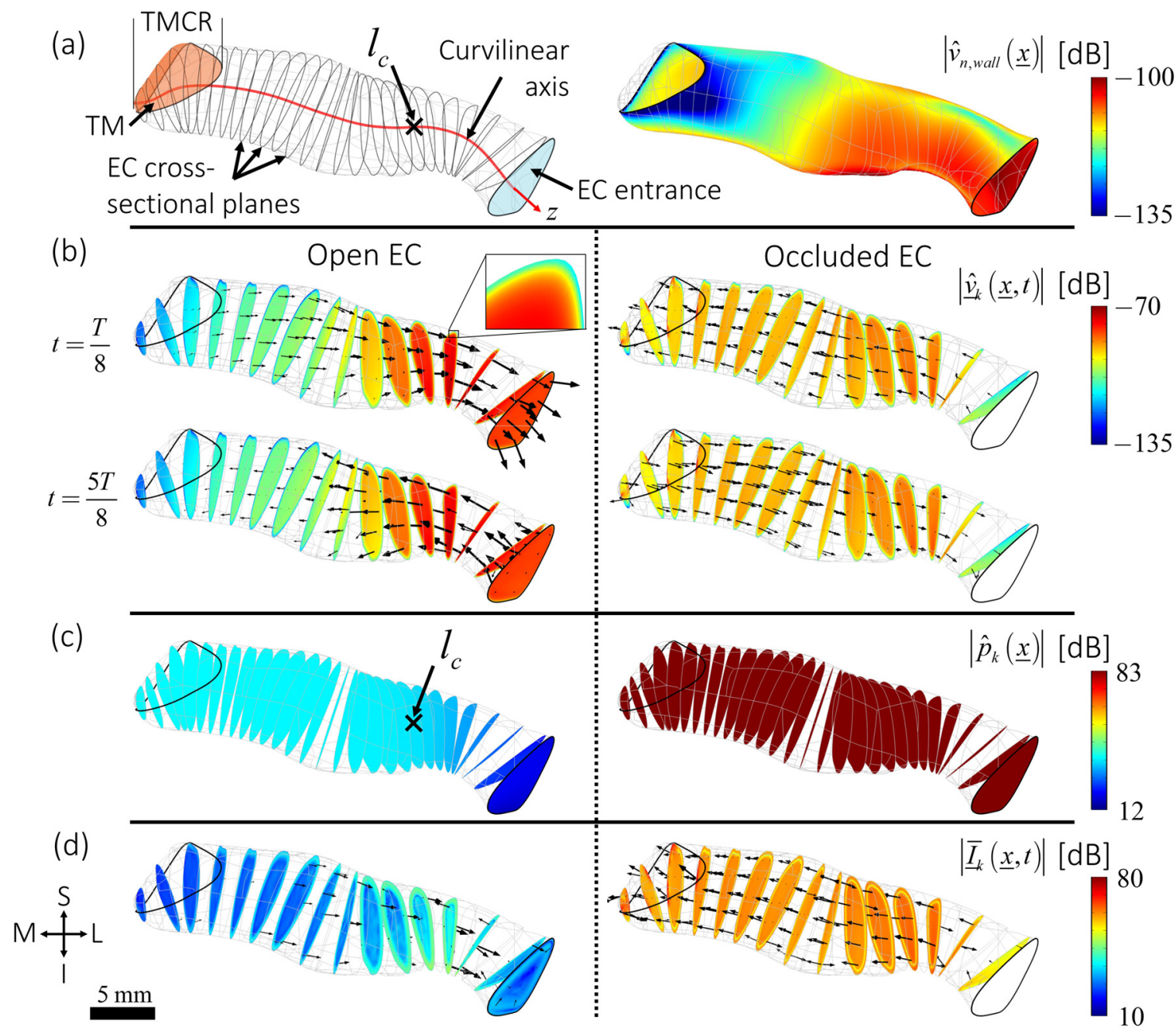

FIG. 4. (Color online) (a) EC wall normal velocity centroid position $l_{c}$ (left) and amplitude $\left|\hat{v}_{n, w a l l}(\underline{x})\right|$ (right) in dB (factor $20, v_{\text {ref }}=1 \mathrm{~m} \mathrm{~s}^{-1}$ ), (b) instantaneous acoustic particle velocity vectors $\underline{v}_{k}(\underline{x}, t)$ (black arrows) and amplitude $\left|\underline{v}_{k}(\underline{x}, t)\right|$ in $\mathrm{dB}$ (colormaps), (c) acoustic pressure level in $\mathrm{dB}$ (factor 20 , $p_{r e f}=2 \times 10^{-5} \mathrm{~Pa}$ ), and (d) active acoustic intensity vectors $\underline{I}_{k}(\underline{x})$ (black arrows) and amplitude $\left|\underline{I}_{k}(\underline{x})\right|$ (colormaps) in $\mathrm{dB}\left(\right.$ factor $10, I_{r e f}=1 \times 10^{-12} \mathrm{~W} \mathrm{~m}{ }^{-2}$ ) computed at $100 \mathrm{~Hz}$ using the coupled elasto-acoustic (visco-thermal) FE model. The white EC entrance surface in (b) and (d) corresponds to zero value.

side). In the occluded case, the volume velocity passing through the $\mathrm{TM}$ is $\approx 6$ to $45 \mathrm{~dB}$ larger than in the open case: the occlusion drastically increases the volume velocity transferred between the EC wall and the TM. This volume velocity is however $\approx 7 \mathrm{~dB}$ lower than that passing through the

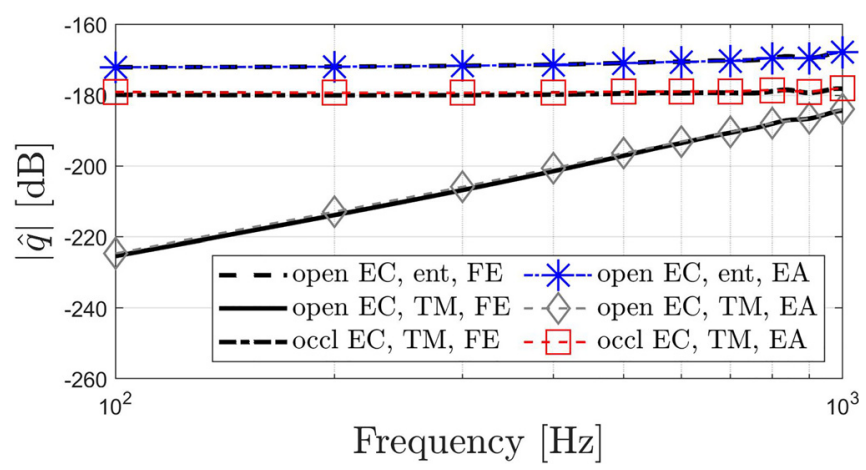

FIG. 5. (Color online) Level in $\mathrm{dB}$ (factor $20, q_{\text {ref }}=1 \mathrm{~m}^{3} \mathrm{~s}^{-1}$ ) of the volume velocity passing through the EC entrance (zero in the occluded case) and the TM computed using both FE and EA models. open EC entrance due to the local volume changes that result from the compression of the air within the occluded EC.

\section{Acoustic impedance of the earcanal cavity}

The EC cavity acoustic impedance governs the acoustic pressure generated in reaction to the vibration of the EC wall. Based on EA analogy, the EC cavity acoustic impedance represents the "opposition" of the EC cavity to the transfer of the volume velocity imposed by its wall. This "opposition" is manifested in terms of acoustic pressure. Figure 6(a) displays the level in $\mathrm{dB}$ of the EC cavity acoustic impedance in open and occluded EC computed using both FE and EA models. The occlusion is seen to drastically increase (by $\approx 8$ to $47 \mathrm{~dB}$ ) the EC cavity acoustic impedance. $^{28,29}$ It should be noted that the EC cavity acoustic impedance computed using FE and EA models are not strictly equal. Indeed, the former uses the surface averaged EC wall acoustic pressure [see Eq. (3)] while the latter rather considers the acoustic pressure at the position $l_{c}$ of the volume velocity source Q (see Sec. IIB 2). This has no 


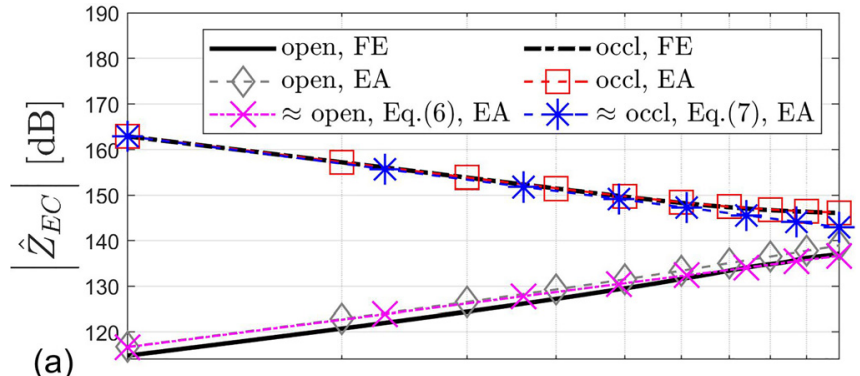

(a)

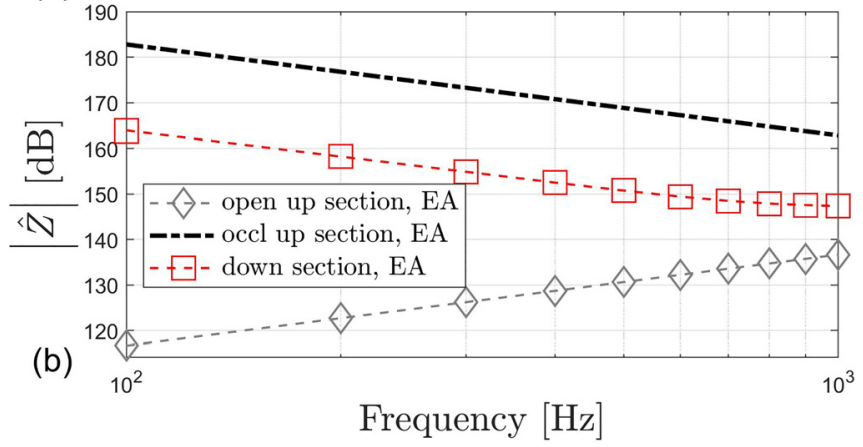

FIG. 6. (Color online) Acoustic impedance levels in $\mathrm{dB}$ (factor 20, $Z_{\text {ref }}=1$ $\mathrm{Ns} \mathrm{m}^{-5}$ ) of (a) the EC cavity seen by its wall (FE model) or the source Q (EA model) and of (b) the upstream and downstream EC sections seen by the source Q (EA model) in both open and occluded cases.

influence in the occluded case since the acoustic pressure is homogenous at LF but explains the difference of $\approx 2 \mathrm{~dB}$ in the open case between FE and EA simulations [see Fig. 6(a)] due to the open EC acoustic pressure gradient [see Fig. 4(c), left side, and Sec. III D].

According to Fig. 6(a), the level of the EC cavity acoustic impedance increases with frequency by $\approx+20 \mathrm{~dB} /$ decade in the open case while it decreases with frequency by $\approx-20 \mathrm{~dB} /$ decade in the occluded case. ${ }^{29}$ From Eq. (6), the EA model indicates that, at LF, the open EC cavity acoustic impedance is approximately mass-controlled $\left(L_{u}+L_{\text {rad }}\right)$ which is proportional to $\omega(+20 \mathrm{~dB} /$ decade $)$. This means that the air in the open EC upstream section is being mainly accelerated at LF. The acoustic mass $L_{u}$ of the open EC upstream section depends on the location of the source $\mathrm{Q}$ which is assumed equal to the curvilinear position $l_{c}$ of the EC wall normal velocity centroid computed using the FE model. The evaluation of this assumption as well as the influence of the distribution of the EC wall vibration on the $\mathrm{OE}$ is presented in Sec. IIIF. From Eq. (7), the EA model indicates that, at LF, the occluded EC cavity acoustic impedance is approximately governed by its whole acoustic compliance which is proportional to $\omega^{-1}$ ( $-20 \mathrm{~dB} /$ decade). This means that the air in the occluded EC is being mainly compressed at LF. According to Fig. 6(a), neglecting the influence of the downstream section, including the TM, in the open case (dashed-dotted purple curve with crosses) or simplifying the TM to an equivalent compliance $C_{T M}$ in the occluded case (dashed blue curve with stars) leads to an underestimation of $\approx 2-4 \mathrm{~dB}$ at $1 \mathrm{kHz}$.

The EA model is now used to provide simple interpretations of the volume velocity transfer in the EC cavity (see
Sec. III B). The source $\mathrm{Q}$ representing the EC wall schematically divides the EC cavity into the upstream and downstream sections towards the EC entrance and the TM, respectively [see Fig. 3(a)]. Acoustic impedance levels of these sections are displayed in Fig. 6(b). In the open case, the EC upstream section has an impedance $\approx 11-47 \mathrm{~dB}$ lower than the downstream section which is almost seen as infinite. The volume velocity is transferred through the "path" of least "opposition" thus between the EC wall and the opening. The mass-controlled open EC upstream section acts as a shunt ${ }^{27}$ for the volume velocity imposed by the EC wall. In the occluded case, the EC upstream section has an impedance rather $\approx 15-19 \mathrm{~dB}$ higher than the downstream section due to the acoustically rigid occlusion. The volume velocity is thus mainly transferred between the EC wall and the TM.

\section{Acoustic pressure in the earcanal cavity}

The level in $\mathrm{dB}$ of the acoustic pressure generated by the EC wall in both open and occluded cases computed at $100 \mathrm{~Hz}$ using the FE model is illustrated in Fig. 4(c). In the open case (left side), an acoustic pressure gradient is observed between the EC opening and approximately the curvilinear position $l_{c}$ of the EC wall normal velocity centroid while the acoustic pressure level is then almost homogeneous up to the TM. According to the EA model, the acoustic pressure level gradient is explained by the acoustic mass $L_{u}$ of the EC upstream section while the acoustic compliance of the EC downstream section is responsible for the acoustic pressure level homogeneity. The distribution of the EC wall vibration as well as the EC shape thus govern the acoustic pressure field induced by $\mathrm{BC}$ stimulation in the open EC. To the authors' knowledge, the acoustic pressure distribution in the open EC has only been studied for acoustic excitations from the surrounding environment ${ }^{50,51}$ or for a reverse mechanical stimulation of the TM from the ossicles. ${ }^{52}$ In the occluded EC, the acoustic pressure level is homogeneous at LF [see Fig. 4(c), right side] since it is controlled by its acoustic compliance. In addition, the acoustic pressure level is higher in the occluded EC compared to the open EC. This observation is corroborated by Fig. 7 which

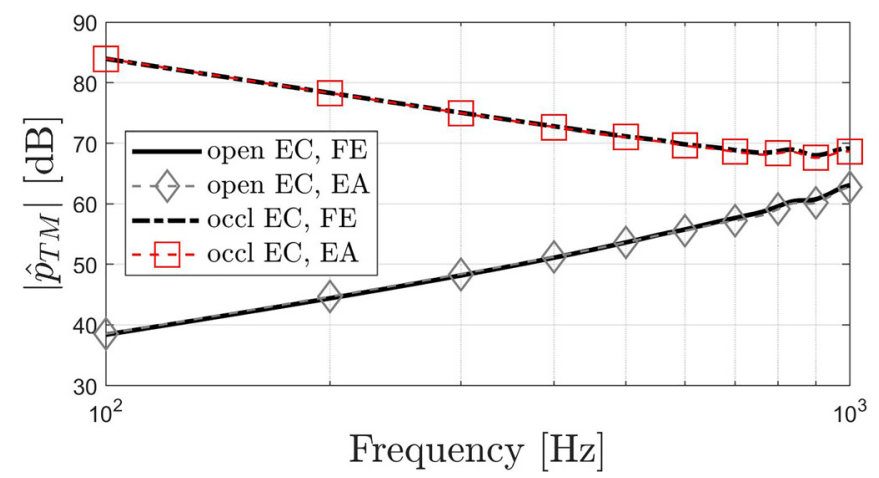

FIG. 7. (Color online) Level in $\mathrm{dB}$ (factor 20 , $p_{\text {ref }}=2 \times 10^{-5} \mathrm{~Pa}$ ) of TM acoustic pressure computed in open and occluded cases using both FE and EA models. 
displays the TM acoustic pressure level computed in both open and occluded cases using FE and EA models. In both cases, the slope of the TM acoustic pressure level is the conjunction of the EC cavity acoustic impedance level and of the volume velocity level imposed by the EC wall. Since the occlusion increases the EC cavity acoustic impedance (see Sec. III C), the acoustic pressure generated by the EC wall is also increased. ${ }^{16,28,29}$ The TM acoustic pressure is the force per unit surface responsible for the motion of the TM. The increase of acoustic pressure at LF leads to the increase of volume velocity at the TM in the occluded case (see Fig. 5).

\section{E. Acoustic intensity and power flow in the earcanal cavity}

The propagation of acoustic waves in the EC is associated with an acoustic power flow. Figure 8 displays the level in $\mathrm{dB}$ of the acoustic power injected into the EC cavity by its wall, computed in open and occluded cases using both FE (LRF) and EA models. Note that the FE (LRF) model compares well to the visco-thermal model computed at $100 \mathrm{~Hz}, 500 \mathrm{~Hz}$, and $1 \mathrm{kHz}$, except that it underestimates by $\approx 1 \mathrm{~dB}$ the acoustic power dissipated by visco-thermal effects in the open EC cavity. According to Fig. 8, the acoustic power injected by the EC wall is significantly higher in the occluded case compared to the open one. ${ }^{30}$ For a given volume velocity imposed to the EC cavity, Eq. (11) of the EA model explicitly indicates that the injected acoustic power is governed by the acoustic resistance of the EC cavity seen by the source Q representing the EC wall. Using the current FE model, an expression similar to Eq. (11) has been proposed by Brummund et al. ${ }^{30}$ but is only equivalent to Eq. (5) if at least the acoustic pressure field or the EC wall normal velocity field is homogeneous over the EC wall surface. The first condition is only valid in the occluded case at LF (see Sec. III D) while the second condition cannot be expected in any case (see Sec. III A). In the open case, the acoustic pressure gradient thus explains the difference $(<2 \mathrm{~dB})$ seen in Fig. 8 between FE and EA simulations while results in the occluded case are in good agreement. Brummund et al. have shown that the EC cavity acoustic

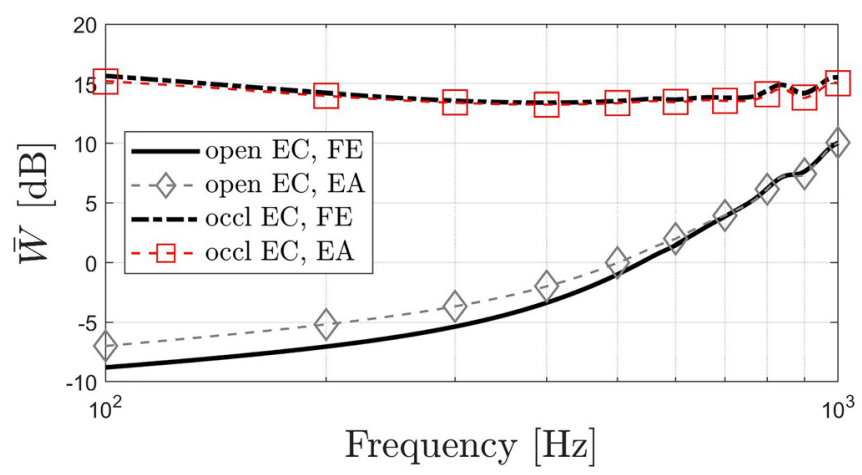

FIG. 8. (Color online) Level in $\mathrm{dB}$ (factor $10, W_{\text {ref }}=1 \times 10^{-12} \mathrm{~W}$ ) of the time averaged acoustic power injected per unit time into the EC cavity by its wall (FE model) and the source Q (EA model) computed in open and occluded cases. resistance drastically increases due to the occlusion ${ }^{30}$ but have not accounted for visco-thermal dissipation in the EC cavity. The increase of the EC cavity acoustic resistance due to the occlusion is also found in this work (not shown here). This is coherent with the increase of the acoustic power injected to the occluded EC cavity (see Fig. 8). However, neglecting the visco-thermal dissipation underestimates the open EC cavity acoustic resistance by $\approx 42 \mathrm{~dB}$ at $100 \mathrm{~Hz}$ (and $\approx 1 \mathrm{~dB}$ at $1 \mathrm{kHz}$ ) which consequently underestimates the acoustic power injected in the open $\mathrm{EC}$ by $\approx 21 \mathrm{~dB}$ at $100 \mathrm{~Hz}$ (and $\approx 0.5 \mathrm{~dB}$ at $1 \mathrm{kHz}$ ). In the occluded case, neglecting the visco-thermal losses slightly underestimates the occluded EC acoustic resistance $(<1.5 \mathrm{~dB})$ and the acoustic power injected $(<1 \mathrm{~dB})$ because the dissipation at the TM dominates (see Appendix D). Accounting for viscothermal losses in the EC cavity is thus necessary to study the acoustic power flow illustrated in Fig. 4(d) and to accurately compute the acoustic power balance, in particular in the open case, ${ }^{33}$ detailed in Appendix D. However, viscothermal losses do not significantly influence the acoustic pressure nor the volume velocity transfer in the EC cavity at LF. Indeed, as shown in Sec. III C using the EA model, the vibro-acoustic behavior of the EC cavity can be well approximated at LF using acoustic reactances only. The use of the acoustic power balance approach to analyze and interpret the $\mathrm{OE}^{30}$ is thus debatable (see Sec. IV). The increase of acoustic power dissipated at the TM due to the occlusion $^{30}$ (not shown here) is simply explained by the increased volume velocity transferred through the TM (see Sec. III B).

\section{F. Occlusion effect}

Figure 9 displays the OE computed using both FE (solid black curve) and EA (dashed gray curve with diamonds) models. The $\mathrm{OE}$ begins at $\approx 45 \mathrm{~dB}$ at $100 \mathrm{~Hz}$ and decreases with frequency by $\approx-40 \mathrm{~dB} /$ decade. Similar slopes are observed with other models at $\mathrm{LF}^{16,28,31,32}$ while the amplitude obtained here corresponds to an extreme case of shallow insertion due to the occlusion at the EC entrance. ${ }^{1,16}$ Experimentally, at frequencies less than $\approx 300 \mathrm{~Hz}$, the $\mathrm{OE}$ is often less than that predicted by Fig. 9, possibly due to an

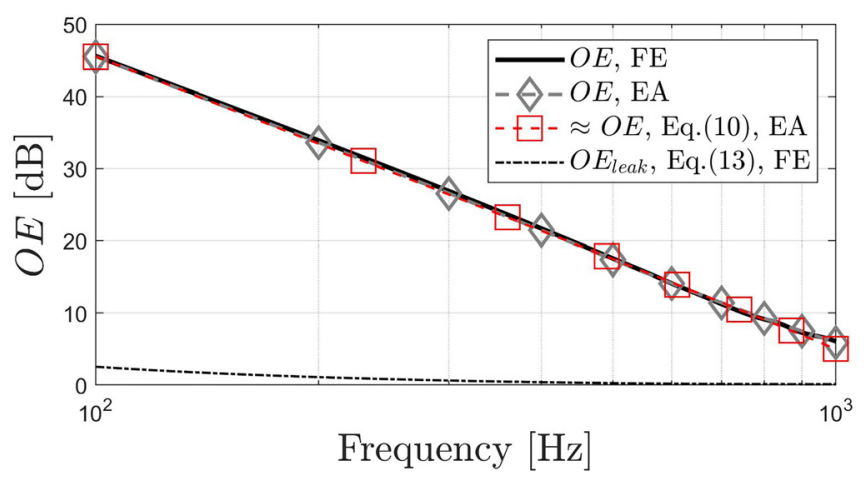

FIG. 9. (Color online) OE computed using FE and EA models. $O E_{\text {leak }}$ refers to a hypothetical OE detailed in Sec. IV. 
incomplete seal between the occlusion device and the EC wall. $^{28,30}$

Figure 9 also displays the LF approximation of the OE (dashed red curve with squares) computed from Eq. (10) of the EA model. From Eq. (10), the slope of $-40 \mathrm{~dB} /$ decade of the $\mathrm{OE}$ is completely described by the dependence on $\omega^{-2}$. This dependence is explained by the change in the character of the impedance between the mass-controlled $\left(L_{\text {rad }}+L_{u}\right)$ open EC state and the compliance-controlled $\left(C_{u}+C_{d}+C_{T M}\right)$ occluded EC state. As shown in Fig. 6(a), neglecting the influence of the EC downstream section, including the TM, in the open case or simplifying the TM to an equivalent compliance $C_{T M}$ in the occluded case, leads to inaccuracies in the computation of the EC cavity acoustic impedance as the frequency increases. In the $\mathrm{OE}$ computed using Eq. (10), these inaccuracies approximately compensate.

In the EA model, the acoustic mass $L_{u}$ of the open EC depends on the position of the volume velocity source Q. This position is generally assumed or adjusted based on sound pressure measurements in the EC cavity. In this work, the source is presumed to be concentrated at the curvilinear position $l_{c}$ (defined from the TM) of the EC wall normal velocity computed using the FE model. This assumption and the influence of the EC wall vibration distribution on the $\mathrm{OE}$ are now evaluated. OE induced by a volume velocity source while the source position $l_{c}$ varies from the TMCR to the $\mathrm{EC}$ entrance (following the EC curvilinear axis) in both FE (EC cavity only) and EA models are computed at $100 \mathrm{~Hz}$ and displayed in Fig. 10. The OE increases with the curvilinear position $l_{c}$ because the acoustic mass $L_{u}$ of the open EC decreases "proportionally" (depending on the EC shape). The whole acoustic compliance $\left(C_{u}+C_{d}+C_{T M}\right)$ of the occluded EC remains constant when $l_{c}$ varies. EA simulation is in good agreement with FE one because both models share the same EC shape. Indeed, note that using a uniform EC cylindrical duct of same volume and EC wall surface leads to significant discrepancies (up to $\approx 6 \mathrm{~dB}$ in the constriction region, not shown here). Figure 10 also displays the OE computed at $100 \mathrm{~Hz}$ using the coupled FE model for

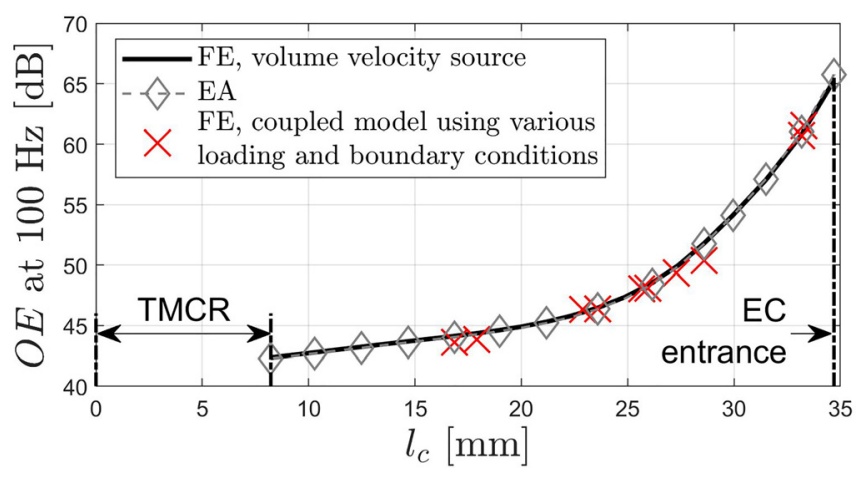

FIG. 10. (Color online) OE computed at $100 \mathrm{~Hz}$ as a function of the curvilinear position $l_{c}$ of the volume velocity source (FE with the EC cavity only and EA models) and of the EC wall normal velocity centroid (coupled FE model using various loading and boundary conditions summarized in Appendix B). several EC wall vibration distributions induced by various sets of loading and boundary conditions presented in Appendix B and characterized by the curvilinear centroid position $l_{c}$. Results show an accurate correspondence between the $\mathrm{OE}$ induced by various EC wall vibration distributions characterized by their centroid position $l_{c}$ (red crosses) and by an equivalent volume velocity source located at the same position $l_{c}$. Differences of $\approx 0.5$ and 1 $\mathrm{dB}$ are observed around $\approx 17$ and $29 \mathrm{~mm}$ possibly due to an influence of the shape of the EC wall vibration distribution which is only characterized here in a simplified way by the centroid position. According to Fig. 10, the influence of the $\mathrm{EC}$ wall vibration distribution on the $\mathrm{OE}$ is mainly observed in the EC cartilaginous part close to the EC entrance where the $\mathrm{OE}$ increases by $\approx 20 \mathrm{~dB}$ from 20 to $34.7 \mathrm{~mm}$. This distribution would be expected to vary with different stimulus types and locations. Such variation might explain the variation in $\mathrm{OE}$ with stimulator position ${ }^{53}$ and could also contribute to the OE inter-individual difference. ${ }^{16}$ Furthermore, this distribution could be frequency-dependent possibly due to EC structural modes which are not observed using the current truncated FE model (see Sec. III A) but could appear in an entire head model. Such dependence might explain that experimental $\mathrm{OE}$ do not precisely follow the decrease with frequency by $\approx-40 \mathrm{~dB} /$ decade since $L_{u}$ would be frequency-dependent in Eq. (10). Finally, it should be emphasized that the EC wall vibration distribution only influences the open EC here. However, this distribution is also expected to influence the vibro-acoustic behavior of the EC occluded by a real physical occlusion device rather than the current infinite impedance. In the authors' opinion, the decrease of the $\mathrm{OE}$ with the insertion depth is underestimated by the FE model of Brummund et al. ${ }^{30}(\approx 7 \mathrm{~dB}$ lower from 7 to $22 \mathrm{~mm}$ at $100 \mathrm{~Hz}$ ) compared to measurement data $^{16}$ (median values $\approx 15 \mathrm{~dB}$ lower from $\approx 7$ to $22 \mathrm{~mm}$ around $160 \mathrm{~Hz}$ ) because the contribution of the EC bony part on the acoustic pressure generated in the EC cavity is overestimated since the EC wall vibration distribution is concentrated at the mid-length of the EC cavity (see Appendix B) rather than in the cartilaginous part.

\section{INTERPRETATIONS OF THE OCCLUSION EFFECT}

As mentioned in Sec. I, the OE is commonly interpreted as follows: the "sound pressure, vibration, energy, or wave" is presumed to "leak" through the EC opening and to be "trapped" in the occluded EC. A common definition of the verb "leak" states that something (matter or energy) comes out of a container through a hole (the leak). ${ }^{54}$ These interpretations are now discussed.

The EC wall acts as an ideal source of volume velocity (see Sec. III A). This volume velocity is mainly transferred between the EC wall and the EC entrance in the open case or between the EC wall and the TM in the occluded one (see Sec. III B). In harmonic regime, the volume velocity is transferred back and forth and alternatively changes sign. The volume velocity cannot literally "leak" (even through 
the EC opening) since it comes in and out. Qualifying the volume velocity transfer between the wall and the entrance as a "leak" and that between the wall and the TM as a "trap" may provide a "mental image"16 of these transfers. However, in the authors" opinion, the terms "leak" and "trap" do not accurately represent the physics. In both open and occluded cases, the volume velocity is mainly transferred through the "path" of least "opposition" (see Sec. III C). The term "opposition" is here preferred instead of "resistance"19 because the latter rather refers to the real part of the acoustic impedance.

The acoustic impedance of the EC cavity represents its "opposition" to the volume velocity transfer and governs its reaction in terms of acoustic pressure. The acoustic pressure does not "leak" nor is it "trapped." It simply increases because the "opposition" increases due to the occlusion (see Sec. III D).

From a vibro-acoustic point of view, acoustic pressure and acoustic particle velocity are physical variables that describe at every position and anytime the continuum acoustic field made of "acoustic particles" which oscillate around their rest position (see Sec. III B). In this way, only acoustic waves travel and could literally "leak." A "leak" could be interpreted as acoustic waves not being or being little reflected while a "trap" would mean a high or perfect reflection. Assuming acoustic plane wave propagation in the EC cavity at LF, the normal incidence pressure reflection coefficient $\hat{R}_{i, k}$ is defined ${ }^{47}$ by

$$
\hat{R}_{i, k}=\frac{\hat{Z}_{i, k}-\rho_{0} c_{0} / S_{i}}{\hat{Z}_{i, k}+\rho_{0} c_{0} / S_{i}},
$$

with $\hat{Z}_{i, k}, i \in\{$ ent, $T M\}$, the acoustic impedance of the EC entrance or the TM seen from the EC cavity and $\rho_{0} c_{0}$ the characteristic impedance of the air. Figure 11 displays $\hat{R}_{i, k}$ in (a) modulus and (b) phase. In the LF regime, the modulus of the EC opening reflection coefficient is close to the acoustically rigid occluded EC entrance and higher than the TM. The EC opening is thus rather a "trap" than a "leak" for acoustic waves at LF which are almost entirely reflected due to the impedance mismatch between the open EC cavity and

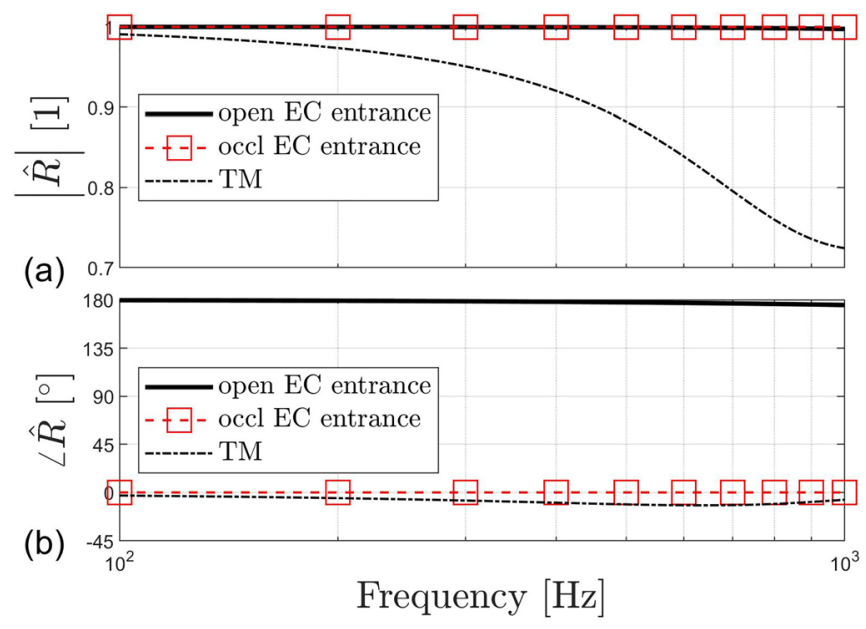

FIG. 11. (Color online) Reflection coefficient of the EC entrance (open and occluded) and the TM in (a) modulus and (b) phase. the surrounding environment just like in the occluded case due to the acoustically rigid termination. However, the change of phase between open and occluded cases [see Fig. 11(b)] influences the way how acoustic waves are reflected at the EC entrance and thus governs the multiple acoustic wave reflections pattern inside the EC cavity. At LF, the occlusion does not "trap" acoustic waves since they are already mainly "trapped" in the open EC cavity but rather influences the way how acoustic waves are "trapped."

However, the reflection coefficient of the EC entrance is not exactly equal to unity in the open case. In harmonic regime, a fraction of time averaged injected acoustic power is radiated through the EC opening in the surrounding environment (see Sec. IIIE). In the case of a rigid occlusion, no acoustic power is radiated through the EC entrance. The question then arises: does the reduction, from $\bar{W}_{\text {ent,open }}$ to 0 , of the acoustic power radiated at the EC entrance (i.e., the seal of the acoustic power "leak") explain the OE? To answer this question, the following hypothetical situation is considered: the occlusion only implies that the acoustic power radiated at the open EC entrance is now dissipated at the TM assuming an equal injected acoustic power in both open and occluded configurations. The resulting $\mathrm{OE}$ is denoted $O E_{\text {leak }}$. At LF, the OE can be expressed in terms of acoustic power dissipated at the TM because the acoustic pressure field is homogeneous over the TM surface. Based on the open EC cavity acoustic power balance, $O E_{\text {leak }}$ is defined by

$$
O E_{\text {leak }}=10 \log _{10}\left(1+\bar{W}_{\text {ent,open }} / \bar{W}_{T M, \text { open }}\right) .
$$

According to Fig. 9, $O E_{\text {leak }}$ (dashed-dotted curve) is negligible which means that the reduction of the acoustic power radiated at the EC entrance cannot cause the OE, as suggested by Brummund et $a l .{ }^{30}$ Indeed, the acoustic power radiated at the EC opening is significantly lower than the increase of acoustic power dissipated at the TM due to the occlusion because the EC wall is not an ideal source of acoustic power (see Sec. IIIE). An interpretation in terms of acoustic power "leak" could be better adapted in the case of constant input acoustic power in open and occluded cases (e.g., insertion loss induced by an EP for an external acoustic excitation). Moreover, the $\mathrm{OE}$ is well explained without accounting for acoustic losses at LF (see Sec. IIIF). Interpreting the OE in terms of acoustic power thus departs from its fundamental mechanism.

In the authors' opinion, the aforementioned common interpretations of the OE misrepresent the underlying physical phenomena. Since the role of the EC cavity acoustic impedance is useful to explain the fundamental mechanism of the $\mathrm{OE}^{16,28,29}$ an interpretation based on this concept is found more accurate. For example, the occlusion increases the "opposition" of the EC cavity to the volume velocity imposed by its wall and increases the amplitude of the acoustic pressure that is generated in reaction, leading to the OE. In the case of hearing aids, the open-fitting decreases this "opposition" and thus the OE. In the case of EP, an incomplete seal has a similar effect at frequencies lower than the Helmholtz resonance formed by the system: the 
neck of the resonator corresponding to the incomplete seal at the EP/EC wall interface and the resonator cavity being the partially occluded EC. ${ }^{28,32,55}$ In the general case, the deep-fitting reduces the $\mathrm{OE}$ because the volume velocity imposed by the EC wall to the occluded EC cavity decreases since the surface as well as the vibration amplitude of the remaining $\mathrm{EC}$ wall diminish with the insertion depth. ${ }^{29}$

\section{CONCLUSION}

The physics of the OE has been thoroughly revisited at LF using a 3D FE model of an outer ear in conjunction with an associated EA model. This study has analyzed and illustrated the EC wall vibration, the transfer of volume velocity imposed by the EC wall, the "opposition" of the EC cavity to this transfer, the acoustic pressure generated in reaction, and the associated acoustic power flow in the EC cavity open and occluded by an infinite impedance. In particular, the distribution of the EC wall vibration is shown to influence the acoustic mass of the open EC cavity seen by its vibrating wall. The EC wall vibration distribution has been characterized by its curvilinear centroid position which has also been assumed as the location of the volume velocity source in the EA model. This assumption has been successfully evaluated for several EC wall vibration patterns induced by various sets of loading and boundary conditions applied to the FE model. In the harmonic regime, the volume velocity is alternatively transferred in the EC cavity through the "path" of least "opposition" (i.e., acoustic impedance), between the source and the EC entrance in the open case, or between the source and the TM in the occluded case. The increase of acoustic pressure is responsible for the increase of TM volume velocity in the occluded case at LF. Common interpretations of the OE in terms of "leak" and "trap" have been shown to misrepresent the fundamental mechanism of the OE related to the EC impedance change. Interpretations based on this concept are considered more accurate. For example, the occlusion increases the "opposition" of the EC cavity to the volume velocity imposed by its wall and thus increases the acoustic pressure generated in reaction, leading to the $\mathrm{OE}$. In this study, the distribution of the EC wall normal velocity has been shown to greatly influence the vibro-acoustic behavior of the open EC (and thus the OE) while that of the occluded EC is not affected due to the use of an acoustically rigid occlusion defined at the EC entrance. For future work, the intricate influence of the occlusion device, in conjunction with the EC wall vibration, on the OE, could be investigated. Furthermore, since different EC wall vibration distributions can share the same centroid, the influence of their shapes could be studied.

\section{ACKNOWLEDGMENTS}

The authors acknowledge the support of the Natural Sciences and Engineering Research Council of Canada (NSERC) (funding reference number RGPIN-2016-06795). Also, the anonymous reviewers are gratefully thanked for their critical and wise comments.
TABLE II. Density $\rho$, Young's modulus $E$, Poisson's ratio $\nu$ and structural loss factor $\eta$ of solid domains.

\begin{tabular}{lccll}
\hline \hline Tissue & $\rho\left(\mathrm{kg} \mathrm{m}^{-3}\right)$ & $E(\mathrm{MPa})$ & $\nu(1)$ & $\eta(1)$ \\
\hline Skin & 1100 & 0.5 & 0.4 & 0.1 \\
Soft & 1080 & 7.2 & 0.26 & 0.05 \\
Bone & 1714 & 11316 & 0.3 & 0.01 \\
\hline \hline
\end{tabular}

\section{APPENDIX A: DOMAIN PROPERTIES}

Solid domain properties used in the FE model are summarized in Table II. Air properties used in both FE and EA models are given in Table III. The molecular dissipation is negligible in the EC cavity so the bulk viscosity $\mu_{B}$ is here zero but could be estimated by $\mu_{B}=0.6 \mu$. $^{40}$

\section{APPENDIX B: VARIOUS SETS OF LOADING AND BOUNDARY CONDITIONS}

Various sets of loading and boundary conditions applied to the FE model are summarized in Fig. 12. These sets are used to vary the distribution of the EC wall normal velocity characterized by the corresponding curvilinear centroid position $l_{c}$ defined from the TM. Configurations $(\mathrm{j})$ and $(\mathrm{k})$ are similar to those used by Brummund et al. ${ }^{30}$ In these cases, switching from fixed (blue surface) to free (gray surface) boundary conditions has little influence on the $\mathrm{OE}^{30}$ because the EC wall vibration distribution is also little influenced $\left(l_{c}\right.$ decreases from 17.9 to $\left.16.9 \mathrm{~mm}\right)$.

\section{APPENDIX C: RADIATION ACOUSTIC IMPEDANCE}

The radiation acoustic impedance $\hat{Z}_{\text {rad }}$ defined at the EC opening is equal to that of a baffled circular piston of radius $r_{\text {ent }}=r_{E C}\left(l_{E C}\right)$ (see Fig. 2) coupled to a semi-infinite acoustic domain and is given ${ }^{47}$ by

$$
\hat{Z}_{\text {rad }}=\frac{\rho_{0} c_{0}}{\pi r_{\text {ent }}^{2}}\left(1-\frac{J_{1}\left(2 k_{0} r_{\text {ent }}\right)}{k_{0} r_{\text {ent }}}-j \frac{H_{1}\left(2 k_{0} r_{\text {ent }}\right)}{k_{0} r_{\text {ent }}}\right),
$$

where $J_{1}$ and $H_{1}$ are the first order Bessel and Struve functions, respectively, and $k_{0}=\omega / c_{0}$ is the lossless wavenumber.

\section{APPENDIX D: ACOUSTIC POWER BALANCE}

This section details the various contributions of the acoustic power balance (not shown here for the sake of

TABLE III. Air density $\rho_{0}$, sound speed $c_{0}$, dynamic viscosity $\mu$, thermal conductivity coefficient $\kappa$, ratio of specific heats $\gamma$, and heat capacity at constant pressure $C_{p}$.

\begin{tabular}{lccc}
\hline \hline Property & Value & Property & Value \\
\hline$\rho_{0}\left(\mathrm{~kg} \mathrm{~m}^{3}\right)$ & 1.2 & $\kappa\left(\mathrm{W} \mathrm{m}^{-1} \mathrm{~K}^{-1}\right)$ & 0.025 \\
$c_{0}\left(\mathrm{~m} \mathrm{~s}^{1}\right)$ & 343 & $\gamma(1)$ & 1.4 \\
$\mu(\mathrm{Pa} \mathrm{s})$ & $1.8313 \times 10^{-5}$ & $C_{p}\left(\mathrm{~J} \mathrm{~kg}^{-1} \mathrm{~K}^{-1}\right)$ & $1.0025 \times 10^{3}$ \\
\hline \hline
\end{tabular}




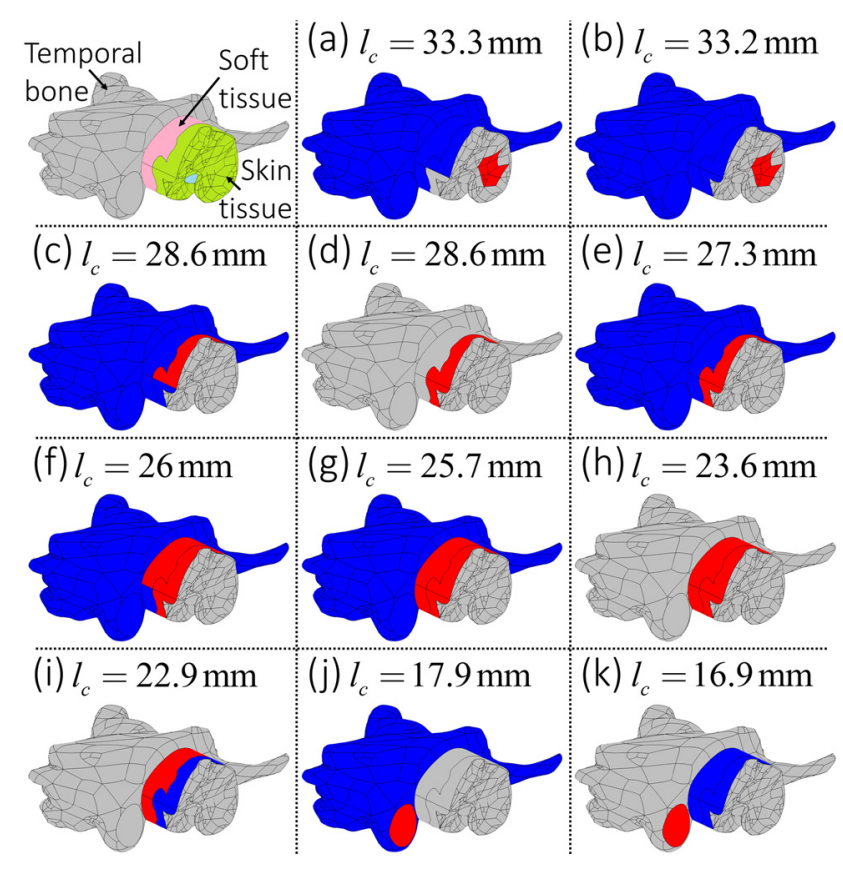

FIG. 12. (Color online) Various sets of mechanical loading and boundary conditions applied to the FE model. Free surface is indicating in gray, fixed surface in blue and excitation surface in red. The curvilinear position $l_{c}$ (from the TM) of the induced EC wall normal velocity centroid is also indicated.

conciseness) in open and occluded cases computed using the LRF 3D FE model. In the open case, the visco-thermal dissipation represents $\approx 99 \%$ at $100 \mathrm{~Hz}$ of the injected acoustic power and decreases with frequency to $\approx 11 \%$ at $1 \mathrm{kHz}$. This dissipation mainly occurs in the EC wall upstream section boundary layer [see Fig. 4(d), left side] where the fluid experiences friction due to its viscosity and the gradient of tangential acoustic particle velocity [see Fig. 4(b), left side]. It should be noted that the damping factor in a real open EC has been estimated to be approximately three times higher than that computed in an EC with smooth wall. ${ }^{39}$ The acoustic power radiated through the EC opening only represents a fraction of the injected acoustic power at LF $(<3 \%)$. This proportion was greatly overestimated by Brummund et al. ${ }^{30}$ since they neglected the visco-thermal dissipation. The remaining proportion of the acoustic power injected to the open EC cavity by its wall is dissipated at the TM and increases with frequency. In the occluded case, the acoustic power dissipated at the TM represents $\approx 85 \%$ at $100 \mathrm{~Hz}$ of the injected acoustic power and increases with frequency to $\approx 99.9 \%$ at $1 \mathrm{kHz}$. The remaining acoustic power injected in the occluded EC is mainly dissipated by thermal effect in the EC wall boundary layer [see Fig. 4(d), right side].

${ }^{1}$ E. Berger, L. Royster, J. Royster, D. Driscoll, and M. Layne, The Noise Manual, 5th ed. (AIHA, Fairfax, VA, 2003).

${ }^{2}$ O. Doutres, F. Sgard, J. Terroir, N. Perrin, C. Jolly, C. Gauvin, and A. Negrini, "A critical review of the literature on comfort of hearing protection devices: Definition of comfort and identification of its main attributes for earplug types,” Int. J. Audio. 58, 824-833 (2019).

${ }^{3}$ S. Kochkin, "MarkeTrak VIII: Consumer satisfaction with hearing aids is slowly increasing," Hear. J. 63(1), 19-20 (2010).
${ }^{4}$ A. Winkler, M. Latzel, and I. Holube, "Open versus closed hearing-aid fittings: A literature review of both fitting approaches," Trends Hear. 20, 1-13 (2016).

${ }^{5}$ M. C. Killion, L. A. Wilber, and G. I. Gudmundsen, "Zwislocki was right," Hear. Instrum. 39(1), 14-18 (1988).

${ }^{6}$ J. Kiessling, B. Brenner, C. Thunberg Jespersen, J. Groth, and O. D. Jensen, "Occlusion effect of earmolds with different venting systems," J. Am. Acad. Audiol. 16(4), 237-249 (2005).

C. Nielsen and S. Darkner, "The cartilage bone junction and its implication for deep canal hearing instrument fittings," Hear. J. 64(3), 35-36 (2011).

${ }^{8}$ S. Stenfelt and R. L. Goode, "Bone-conducted sound: Physiological and clinical aspects," Otol. Neurotol. 26(6), 1245-1261 (2005).

${ }^{9}$ I. Dobrev, J. H. Sim, S. Stenfelt, S. Ihrle, R. Gerig, F. Pfiffner, A. Eiber, A. M. Huber, and C. Röösli, "Sound wave propagation on the human skull surface with bone conduction stimulation," Hear. Res. 355, 1-13 (2017).

${ }^{10}$ H. Sohmer, "Soft tissue conduction: Review, mechanisms, and implications," Trends Hear. 21, 1-8 (2017).

${ }^{11}$ S. Chordekar, R. Perez, C. Adelman, H. Sohmer, and L. Kishon-Rabin, "Does hearing in response to soft-tissue stimulation involve skull vibrations? A within-subject comparison between skull vibration magnitudes and hearing thresholds," Hear. Res. 364, 59-67 (2018).

${ }^{12}$ S. Stenfelt, T. Wild, N. Hato, and R. L. Goode, "Factors contributing to bone conduction: The outer ear," J. Acoust. Soc. Am. 113(2), 902-913 (2003).

${ }^{13}$ S. Stenfelt, "Model predictions for bone conduction perception in the human," Hear. Res. 340, 135-143 (2016).

${ }^{14} \mathrm{E}$. H. Huizing, "Bone conduction-the influence of the middle ear," Acta Oto-Laryngol., Suppl. 155, 1-99 (1960).

${ }^{15}$ J. Schröter and C. Poesselt, "The use of acoustical test fixtures for the measurement of hearing protector attenuation. Part II: Modeling the external ear, simulating bone conduction, and comparing test fixture and realear data," J. Acoust. Soc. Am. 80(2), 505-527 (1986).

${ }^{16} \mathrm{~S}$. Stenfelt and S. Reinfeldt, "A model of the occlusion effect with boneconducted stimulation,” Int. J. Audiol. 46(10), 595-608 (2007).

${ }^{17}$ R. Carle, S. Laugesen, and C. Nielsen, "Observations on the relations among occlusion effect, compliance, and vent size," J. Am. Acad. Audiol. 13(1), 25-37 (2002).

${ }^{18}$ G. Keidser, L. Carter, J. Chalupper, and H. Dillon, "Effect of lowfrequency gain and venting effects on the benefit derived from directionality and noise reduction in hearing aids," Int. J. Audiol. 46(10), 554-568 (2007).

${ }^{19}$ W. Staab, M. Dennis, H. C. Schweitzer, and J. E. Weber, "Measuring the occlusion effect in a deep-fitting hearing device," Hear. Rev. 11(13), 44-50 (2004).

${ }^{20}$ W. J. Staab, "Introduction to deep canal principles," Semin. Hear. 17, 3-19 (1996).

${ }^{21}$ F. K. Kuk, "Perceptual consequence of vents in hearing aids," Br. J. Audiol. 25(3), 163-169 (1991).

${ }^{22} \mathrm{H}$. G. Mueller, "CIC hearing aids: What is their impact on the occlusion effect?," Hear. J. 47(11), 29-30 (1994).

${ }^{23}$ H. G. Mueller, K. E. Bright, and J. L. Northern, "Studies of the hearing aid occlusion effect," Semin. Hear. 17, 21-31 (1996).

${ }^{24} \mathrm{~K}$. Chung, "Challenges and recent developments in hearing aids: Part II. Feedback and occlusion effect reduction strategies, laser shell manufacturing processes, and other signal processing technologies," Trends Amplif. 8(4), 125-164 (2004).

${ }^{25}$ R. C. Borges, M. H. Costa, P. A. Naylor, and A. A. Ferreira, "Impact of the vent size in the feedback-path and occlusion-effect in hearing aids," in 2014 IEEE Biomedical Circuits and Systems Conference (BioCAS) Proceedings (2014), pp. 25-28.

${ }^{26}$ M. O. Hansen and M. R. Stinson, "Air conducted and body conducted sound produced by own voice," Can. Acoust. 26(2), 11-19 (1998).

${ }^{27} \mathrm{~J}$. Tonndorf, "Bone conduction studies in experimental animals," Acta Oto-Laryngol. 213, 1-132 (1966).

${ }^{28}$ M. Ø. Hansen, T. Poulsen, and P. Lundh, "Occlusion effects, Part II: A study of the occlusion effect mechanism and the influence of the earmould properties," Report, Technical University Denmark, 1998.

${ }^{29}$ T. Zurbrügg, A. Stirnemannn, M. Kuster, and H. Lissek, "Investigations on the physical factors influencing the ear canal occlusion effect caused by hearing aids," Acta Acust. Acust. 100(3), 527-536 (2014). 
${ }^{30}$ M. K. Brummund, F. Sgard, Y. Petit, and F. Laville, "Three-dimensional finite element modeling of the human external ear: Simulation study of the bone conduction occlusion effect," J. Acoust. Soc. Am. 135(3), 1433-1444 (2014).

${ }^{31}$ M. K. Brummund, F. Sgard, Y. Petit, F. Laville, and H. Nélisse, "An axisymmetric finite element model to study the earplug contribution to the bone conduction occlusion effect," Acta Acust. Acust. 101(4), 775-788 (2015).

${ }^{32}$ F. Sgard, K. Carillo, and O. Doutres, "A 2d axisymmetric finite element model to assess the contribution of in-ear hearing protection devices to the objective occlusion effect," in Proceedings of Internoise, Madrid, Spain (June 16-19, 2019) .

${ }^{33}$ D. H. Keefe, J. C. Bulen, S. L. Campbell, and E. M. Burns, "Pressure transfer function and absorption cross section from the diffuse field to the human infant ear canal," J. Acoust. Soc. Am. 95(1), 355-371 (1994).

${ }^{34}$ K. Lee and J. G. Casali, "Investigation of the auditory occlusion effect with implications for hearing protection and hearing aid design," in Proceedings of the Human Factors and Ergonomics Society Annual Meeting (SAGE Publications, Los Angeles, CA, 2011), Vol. 55, pp. 1783-1787.

${ }^{35} \mathrm{~K}$. Lee, "Effects of earplug material, insertion depth, and measurement technique on hearing occlusion effect," Ph.D. thesis, Virginia Tech, 2011.

${ }^{36}$ Y. Chang, N. Kim, and S. Stenfelt, "The development of a whole-head human finite-element model for simulation of the transmission of boneconducted sound," J. Acoust. Soc. Am. 140(3), 1635-1651 (2016).

${ }^{37}$ S. Benacchio, O. Doutres, A. Le Troter, A. Varoquaux, E. Wagnac, V. Callot, and F. Sgard, "Estimation of the earcanal displacement field due to in-ear device insertion using a registration method on a human-like artificial ear," Hear. Res. 365, 16-27 (2018).

${ }^{38}$ M. R. Stinson and B. Lawton, "Specification of the geometry of the human ear canal for the prediction of sound-pressure level distribution," J. Acoust. Soc. Am. 85(6), 2492-2503 (1989).

${ }^{39} \mathrm{H}$. Hudde and A. Engel, "Measuring and modeling basic properties of the human middle ear and ear canal. Part II: Ear canal, middle ear cavities, eardrum, and ossicles," Acta Acust. Acust. 84(5), 894-913 (1998).

${ }^{40}$ A. Pierce, Acoustics: An Introduction to its Physical Principles and Applications (Acoustical Society of America, Melville, NY, 1994), pp. 531-534.
${ }^{41}$ W. Kampinga, Y. H. Wijnant, and A. de Boer, "Performance of several viscothermal acoustic finite elements," Acta Acust. Acust. 96(1), 115-124 (2010).

${ }^{42}$ E. Shaw and M. Stinson, "Network concepts and energy flow in the human middle-ear," Acoust. Soc. Am. 69(S1), S43 (1981).

${ }^{43}$ E. Shaw and M. Stinson, "The human external and middle ear: Models and concepts," in Mechanics of Hearing (Springer, New York, 1983), pp. 3-10.

${ }^{44} \mathrm{H}$. Hudde and A. Engel, "Measuring and modeling basic properties of the human middle ear and ear canal. Part III: Eardrum impedances, transfer functions and model calculations," Acta Acust. Acust. 84(6), 1091-1108 (1998).

${ }^{45}$ W. Kampinga, "Viscothermal acoustics using finite elements - analysis tools for engineers," Ph.D. thesis, University of Twente, Netherlands, 2010.

${ }^{46}$ N. Atalla and F. Sgard, Finite Element and Boundary Methods in Structural Acoustics and Vibration (CRC Press, Boca Raton, FL, 2015).

${ }^{47}$ M. Bruneau, Fundamentals of Acoustics (Wiley, New York, 2013).

${ }^{48}$ C. E. Stepp and S. E. Voss, "Acoustics of the human middle-ear air space," The J. Acoust. Soc. Am. 118(2), 861-871 (2005).

${ }^{49}$ K. Carillo, O. Doutres, and F. Sgard, "Numerical investigation of the fundamental low frequency mechanism of the objective occlusion effect: Focus on the earcanal wall vibration," in Proceedings of the ICSV26, Montreal, Quebec, Canada (2019), pp. 1-8.

${ }^{50}$ M. R. Stinson and G. A. Daigle, "Comparison of an analytic horn equation approach and a boundary element method for the calculation of sound fields in the human ear canal," J. Acoust. Soc. Am. 118(4), 2405-2411 (2005).

${ }^{51}$ H. Hudde and S. Schmidt, "Sound fields in generally shaped curved ear canals," J. Acoust. Soc. Am. 125(5), 3146-3157 (2009).

${ }^{52}$ M. E. Ravicz, J. T. Cheng, and J. J. Rosowski, "Sound pressure distribution within human ear canals: II. reverse mechanical stimulation," J. Acoust. Soc. Am. 145(3), 1569-1583 (2019).

${ }^{53}$ S. Reinfeldt, S. Stenfelt, and B. Håkansson, "Estimation of bone conduction skull transmission by hearing thresholds and ear-canal sound pressure," Hear. Res. 299, 19-28 (2013).

${ }^{54}$ See the Macmillan dictionary (2019) for the definition of the term "leak". https://www.macmillandictionary.com/dictionary/american/leak_1.

${ }^{55} \mathrm{M}$. Brummund, "Study of the occlusion effect induced by an earplug: Numerical modelling and experimental validation," Ph.D. thesis, École de technologie supérieure, Canada, 2014. 